\title{
Isolation of Leptospira spp. from companion dogs who received leptospirosis vaccination presented to the Government Veterinary Hospital, Peradeniya
}

\author{
P Senarathne ${ }^{1}$, T Athapattu ${ }^{2}$, P Rajapakse ${ }^{3}$, U Karunadasa ${ }^{4}$, M Fuward ${ }^{5}$, M Thilakarathne ${ }^{4}$, T Herath ${ }^{1}$, \\ D Muthusinghe $^{6}, \mathrm{~K}$ Yoshimatsu $^{6}, \mathrm{~N}_{\text {Koizumi }}{ }^{7}$, CDGamage $^{1}$
}

Introduction and Objectives: Companion animals can act as asymptomatic carriers of zoonotic infections. Canine leptospirosis is a disease where chronic carrier dogs excrete pathogenic leptospires in their urine intermittently, resulting in environmental contamination and a source of infection for humans. The current study aimed to isolate and characterizeLeptospira spp. from both blood and urine of companion dogs who received the leptospirosis vaccine available in Sri Lanka.

Methods: Blood and urine samples were collected from 20 healthy dogs that were vaccinated properly according to the leptospirosis vaccination schedule practiced in the Government Veterinary Hospital, Peradeniya, Kandy. The samples were centrifuged at $3000 \times \mathrm{g}$ for 10 minutes and the supernatant of urine and plasma were separated. Collected urine supernatants were filtered using $0.22 \mu \mathrm{m}$ filters and each filter paper was separately placed in $5 \mathrm{ml}$ of liquid Ellinghausen-McCullough-Johnson-Harris medium with selected antibiotics. Two hundred microliter of plasma was directly inoculated in the same culture media. After confirming the presence of spirochetes in cultures using dark-field microscope under $200 \times$ magnifications, $500 \mu \mathrm{l}$ of the positive culture was centrifuged at $15000 \times \mathrm{g}$ for 20 minutes and DNA were extracted from the pellets. DNA extracts were subjected to two different nested PCRs targeting flaB gene using two different primer sets to identify the pathogenic Leptospira spp. belonging to P1 and P2 subclades, respectively. All PCR positive samples were sequenced, and a phylogenetic tree was constructed including 25 reference strains.

Results: Four of the 20 urine samples, and two of the 20 blood samples yielded spirochetelike organisms. Three of the four urine cultures and one of the two blood cultures gave positive results for PCR,confirming the excretion of live leptospires in their urine. DNA sequencing revealed that the isolates were $L$. interrogans and $L$. kmetyiof P1 subclade, and $L$. licerasiaeof P2 subclade.

Conclusions:A portion of companion dogs who are vaccinated for leptospirosis excrete live pathogenic Leptospira spp.

Keywords: Canine leptospirosis, phylogenetic tree,spirochetes, vaccination

\footnotetext{
${ }^{1}$ Department of Microbiology, Faculty of Medicine, University of Peradeniya, Sri Lanka

${ }^{2}$ Department of Veterinary Public Health and Pharmacology Faculty of Veterinary Medicine and Animal

Science, University of Peradeniya, Sri Lanka

${ }^{3}$ Department of Veterinary Clinical Sciences, Faculty of Veterinary Medicine and Animal Science,

University of Peradeniya, Sri Lanka

${ }^{4}$ Postgraduate Institute of Science, University of Peradeniya, Sri Lanka

${ }^{5}$ Government Veterinary Hospital, Peradeniya, Sri Lanka

${ }^{6}$ Graduate School of Infectious Diseases, Hokkaido University, Sapporo, Japan

${ }^{7}$ Department of Bacteriology I, National Institute of Infectious Diseases, Tokyo, Japan
}

Address for correspondence: Prof C Gamage. Telephone: +94771661460

Email: chandika.gamage@med.pdn.ac.lk (1) https://orcid.org/0000-0003-0974-5730 